\title{
New Generic Attacks against Hash-Based MACs
}

\author{
Gaëtan Leurent ${ }^{1}$, Thomas Peyrin² ${ }^{2}$ and Lei Wang ${ }^{2}$ \\ 1 Université Catholique de Louvain, Belgium \\ gaetan.leurent@uclouvain. be \\ 2 Nanyang Technological University, Singapore \\ thomas.peyrin@gmail.com, wang.lei@ntu.edu.sg
}

\begin{abstract}
In this paper we study the security of hash-based MAC algorithms (such as HMAC and NMAC) above the birthday bound. Up to the birthday bound, HMAC and NMAC are proven to be secure under reasonable assumptions on the hash function. On the other hand, if an $n$-bit MAC is built from a hash function with a $l$-bit state $(l \geq n)$, there is a well-known existential forgery attack with complexity $2^{l / 2}$. However, the remaining security after $2^{l / 2}$ computations is not well understood. In particular it is widely assumed that if the underlying hash function is sound, then a generic universal forgery attack should require $2^{n}$ computations and some distinguishing (e.g. distinguishing- $\mathrm{H}$ but not distinguishing- $\mathrm{R}$ ) and state-recovery attacks should also require $2^{l}$ computations (or $2^{k}$ if $k<l)$.

In this work, we show that above the birthday bound, hash-based MACs offer significantly less security than previously believed. Our main result is a generic distinguishing- $\mathrm{H}$ and state-recovery attack against hash-based MACs with a complexity of only $\tilde{O}\left(2^{l / 2}\right)$. In addition, we show a key-recovery attack with complexity $\tilde{O}\left(2^{3 l / 4}\right)$ against HMAC used with a hash functions with an internal checksum, such as GOST. This surprising result shows that the use of a checksum might actually weaken a hash function when used in a MAC. We stress that our attacks are generic, and they are in fact more efficient than some previous attacks proposed on MACs instanciated with concrete hash functions.

We use techniques similar to the cycle-detection technique proposed by Peyrin et al. at Asiacrypt 2012 to attack HMAC in the related-key model. However, our attacks works in the single-key model for both HMAC and NMAC, and without restriction on the key size.
\end{abstract}

Keywords: NMAC, HMAC, hash function, distinguishing-H, key recovery, GOST.

\section{Introduction}

Message Authentication Codes (MACs) are crucial components in many security systems. A MAC is a function that takes a $k$-bit secret key $K$ and an arbitrarily long message $M$ as inputs, and outputs a fixed-length tag of size $n$ bits. The tag is used to authenticate the message, and will be verified by the receiving party using the same key $K$. Common MAC algorithms are built from block 
ciphers (e.g. CBC-MAC), from hash functions (e.g. HMAC), or from universal hash functions (e.g. UMAC). In this paper we study MAC algorithms based on hash functions.

As a cryptographic primitive, a MAC algorithm should meet some security requirements. It should be impossible to recover the secret key except by exhaustive search, and it should be computationally impossible to forge a valid MAC without knowing the secret key, the message being chosen by the attacker (existential forgery) or given as a challenge (universal forgery). In addition, cryptanalysts have also studied security notions based on distinguishing games. Informally, the distinguishing- $\mathrm{R}$ game is to distinguish a MAC construction from a random function, while the distinguishing- $\mathrm{H}$ game is to distinguish a known MAC construction (e.g. HMAC) instantiated with a known component (e.g. SHA-1) under a random key from the same construction instantiated with a random component (e.g. HMAC with a fixed input length random function).

One of the best known MAC algorithm is HMAC [2], designed by Bellare et al. in 1996. HMAC is now widely standardized (by ANSI, IETF, ISO and NIST), and widely deployed, in particular for banking processes or Internet protocols (e.g. SSL, TLS, SSH, IPSec). It is a single-key version of the NMAC construction, the latter being built upon a keyed iterative hash function $H_{K}$, while HMAC uses an unkeyed iterative hash function $H$ :

$$
\begin{aligned}
\operatorname{NMAC}\left(K_{\text {out }}, K_{\text {in }}, M\right) & =H_{K_{\text {out }}}\left(H_{K_{\text {in }}}(M)\right) \\
\operatorname{HMAC}(K, M) & =H(K \oplus \text { opad } \| H(K \oplus \text { ipad } \| M))
\end{aligned}
$$

where opad and ipad are predetermined constants, and where $K_{\text {in }}$ denotes the inner key and $K_{\text {out }}$ the outer one.

More generally, a MAC algorithm based on a hash function uses the key at the beginning and/or at the end of the computation, and updates an $l$-bit state with a compression function. The security of MAC algorithms is an important topic, and both positive and negative results are known. On the one hand, there is a generic attack with complexity $2^{l / 2}$ based on internal collisions and length extension [17. This gives an existential forgery attack, and a distinguishing-H attack. One the other hand, we have security proofs for several MAC algorithms such as HMAC and sandwich-MAC 22126]. Roughly speaking, the proofs show that some MAC algorithms are secure up to the birthday bound $\left(2^{l / 2}\right)$ under various assumptions on the compression function and hash function.

Thanks to those results, one may consider that the security of hash-based MAC algorithms is well understood. However, there is still a strong interest in the security above the birthday bound. In particular, it is very common to expect security $2^{k}$ for key recovery attacks if the hash function is sound; the Encyclopedia of Cryptography and Security article on HMAC states explicitly [16] "A generic key recovery attack requires $2^{n / 2}$ known text-MAC pairs and $2^{n+1}$ time" (assuming $n=l=k$ ). Indeed, key recovery attacks against HMAC with a concrete hash function with complexity between $2^{l / 2}$ and $2^{l}$ have been considered as important results 92427 . Similarly, the best known dinstinguishing-H and state-recovery attacks have a complexity of $2^{l}$ (or $2^{k}$ if $k<l$ ), and distinguishing-H attacks on 
Table 1. Comparison of our generic attacks on HMAC, and some previous attacks on concrete hash function. We measure the complexity as the number of calls to the compression function (i.e. number of messages times message length).

\begin{tabular}{|c|c|c|c|c|}
\hline \multirow{2}{*}{$\frac{\text { Function }}{\text { HMAC-MD5 }}$} & Attack & \multicolumn{2}{|c|}{ Complexity M. len Notes } & Ref \\
\hline & dist-H, state rec. & $2^{97}$ & 2 & 25 \\
\hline HMAC-SHA-O & dist-H & $2^{100}$ & 2 & 12 \\
\hline HMAC-HAVAL (3-pass) & dist-H & $2^{228}$ & 2 & 12 \\
\hline HMAC-SHA-1 (43 first steps) & dist-H & $2^{154}$ & 2 & 12 \\
\hline HMAC-SHA-1 (58 first steps) & dist-H & $2^{158}$ & 2 & 18 \\
\hline HMAC-SHA-1 (61 mid. steps) & dist-H & $2^{100}$ & 2 & 18 \\
\hline HMAC-SHA-1 (62 mid. steps) & dist-H & $2^{157}$ & 2 & 18 \\
\hline \multicolumn{5}{|l|}{ Generic attacks: } \\
\hline \multirow[t]{3}{*}{ hash-based MAC (e.g. HMAC } & dist-H & $O\left(2^{l / 2}\right)$ & $2^{l / 2}$ & Sec. 4 \\
\hline & state rec. & $\tilde{O}\left(2^{l / 2}\right)$ & $2^{l / 2}$ & Sec. 5 \\
\hline & dist-H, state rec. & $O\left(2^{l-s}\right)$ & $s \leq l /$ & full version \\
\hline \multirow[t]{2}{*}{ HMAC with a checksum } & key rec. & $O\left(l \cdot 2^{3 l / 4}\right)$ & $2^{l / 2}$ & Sec. 7 \\
\hline & & $O\left(l \cdot 2^{3 l / 4}\right)$ & $2^{l / 4}$ & full version \\
\hline HMAC-MD5* & dist-H, state rec. & $\begin{array}{l}2^{67}, 2^{78} \\
O\left(2^{96}\right)\end{array}$ & $\begin{array}{l}2^{64} \\
2^{32}\end{array}$ & $\begin{array}{l}\text { Sec. } 45 \\
\text { full version }\end{array}$ \\
\hline HMAC-HAVAL $^{\dagger}$ (any) & dist-H, state rec. & $\mathrm{O}\left(2^{202}\right)$ & $2^{54}$ & full version \\
\hline HMAC-SHA- $1^{\dagger}$ & dist-H, state rec. & $\mathrm{O}\left(2^{120}\right)$ & $2^{40}$ & full version \\
\hline \multirow[t]{2}{*}{ HMAC-GOST ${ }^{\star}$} & key rec. & $2^{200}$ & $2^{128}$ & Sec. 7 \\
\hline & & $2^{200}$ & $2^{64}$ & full version \\
\hline
\end{tabular}

* The MD5 and GOST specifications allow arbitrary-length messages

$\dagger$ The SHA-1 and HAVAL specifications limits the message length to $2^{64}$ bits (and $2^{64}$ bits is $2^{54}$ blocks)

HMAC with a concrete hash function with complexity between $2^{l / 2}$ and $2^{l}$ have been considered as important results $12,18,25]$.

Our Contributions. In this paper we revisit the security of hash-based MAC above the birthday bound. We describe a generic distinguishing- $\mathrm{H}$ attack in the single key model and with complexity of only $O\left(2^{l / 2}\right)$ computations, thus putting an end to the long time belief of the cryptography community that the best generic distinguishing-H on NMAC and HMAC requires $\Omega\left(2^{l}\right)$ operations. Instead, we show that a distinguishing-H attack is not harder than a distinguishing- $\mathrm{R}$ attack. Our results actually invalidate some of the recently published cryptanalysis works on HMAC when instantiated with real hash functions [12 1825]

Our method is based on a cycle length detection, like the work of Peyrin et. al 14, but our utilization is quite different and much less restrictive: instead of iterating many times HMAC with small messages and a special related-key, we will use only a few iterations with very long messages composed of almost the same message blocks to observe the cycle and deduct information from it. Overall, 
unlike in [14, our technique works in the single-key model, for both HMAC and NMAC, and can be applied for any key size. In addition, leveraging our new ideas, we provide a single-key internal state recovery for any hash-based MAC with only $O\left(l \cdot \log (l) \cdot 2^{l / 2}\right)$ computations.

We also introduce a different approach to reduce the length of the queried messages for distinguishing- $\mathrm{H}$ and internal-state-recovery attacks. Due to the limited space, we only give a quick overview of these shorter-message attacks, and refer the reader to the full version of this paper for more details.

Finally, this internal state recovery can be transformed into a single-key key recovery attack on HMAC with complexity $O\left(l \cdot 2^{3 l / 4}\right)$ when instantiated with a hash function using a checksum, such as the GOST hash function [6]. A surprising corollary to our results is that incorporating a checksum to a hash function seems to actually reduce the security of the overall HMAC design.

We give an overview of our results, and a comparison with some previous analysis in Table 1 .

The description of HMAC/NMAC algorithms and their security are given in Section 2 and we recall in Section 3 the cycle-detection ideas from [14. Then, we provide in Section 4 the generic distinguishing- $\mathrm{H}$ attack. We give in Sections 5 an internal state recovery method, and finally describe our results when the hash function incorporates a checksum in Section 7.

\section{Hash-Based MAC Algorithms}

In this paper, we study a category of MAC algorithms based on hash functions, where the key is used at the beginning and at the end of the computation, as described in Figure 1. More precisely, we consider algorithms where: the message processing is done by updating an internal state $x$ using a compression function $h$; the state is initialized with a key dependent value $I_{k}$; and the tag is computed from the last state $x_{p}$ and the key $K$ by an output function $g$.

$$
x_{0}=I_{K} \quad x_{i+1}=h\left(x_{i}, m_{i}\right) \quad \operatorname{MAC}_{K}(M)=g\left(K, x_{p},|M|\right)
$$

In particular, this description covers NMAC/HMAC [2], envelope-MAC [21], and sandwich-MAC 26. The results described in Sections 4 and 5 can be applied to any hash-based MAC, but we focus on HMAC for our explanations because it is the most widely used hash-based MAC, and its security has been widely analyzed already. On the other hand the result of Section 7 is specific to MAC algorithms that process the key as part of the message, such as HMAC.

\subsection{Description of NMAC and HMAC}

A Hash Function. $H$ is a function that takes an arbitrary length input message $M$ and outputs a fixed hash value of size $n$ bits.

Virtually every hash function in use today follows an iterated structure like the classical Merkle-Damgård construction [134]. Iterative hash functions are 


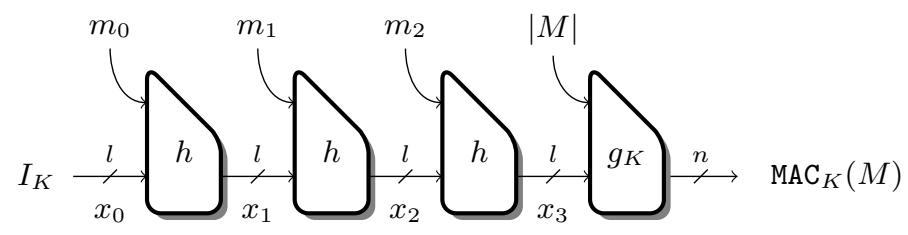

Fig. 1. Hash-based MAC. Only the initial value and the final transformation are keyed.

built upon successive applications of a so-called compression function $h$, that takes a $b$-bit message block and a $l$-bit chaining value as inputs and outputs a $l$-bit value (where $l \geq n$ ). An output function can be included to derive the $n$-bit hash output from the last chaining variable and from the message length. When $l>n$ (resp. when $l=n$ ) we say that the hash function is wide pipe (resp. narrow pipe).

The message $M$ is first padded and then divided into blocks $m_{i}$ of $b$ bits each. Then, the message blocks are successively used to update the $l$-bit internal state $x_{i}$ with the compression function $h$. Once all the message blocks have been processed, the output function $g$ is applied to the last internal state value $x_{p}$.

$$
x_{0}=I V \quad x_{i+1}=h\left(x_{i}, m_{i}\right) \quad \text { hash }=g\left(x_{p},|M|\right)
$$

The MAC algorithm NMAC [2] uses two $l$-bit keys $K_{\text {out }}$ and $K_{\text {in }}$. NMAC replaces the public $I V$ of a hash function $H$ by a secret key $K$ to produce a keyed hash function $H_{K}(M)$. NMAC is then defined as:

$$
\operatorname{NMAC}\left(K_{\text {out }}, K_{\text {in }}, M\right)=H_{K_{\text {out }}}\left(H_{K_{\text {in }}}(M)\right)
$$

The MAC algorithm HMAC [2] is a single-key version of NMAC, with $K_{\text {out }}=$ $h(I V, K \oplus$ opad $)$ and $K_{i n}=h(I V, K \oplus$ ipad $)$, where opad and ipad are $b$-bit constants. However, a very interesting property of HMAC for practical utilization is that it can use any key size and can be instantiated with an unkeyed hash function (like MD5, SHA-1, etc. which have a fixed $I V$ ):

$$
\operatorname{HMAC}(K, M)=H(K \oplus \operatorname{opad} \| H(K \oplus \operatorname{ipad} \| M)) .
$$

where $\|$ denotes the concatenation operation. For simplicity of the description and without loss of generality concerning our attacks, in the rest of this article we assume that the key can fit in one compression function message block, i.e. $k \leq b$ (note that $K$ is actually padded to $b$ bits if $k<b$ ).

\subsection{Security of NMAC and HMAC}

In [1, Bellare proved that the NMAC construction is a pseudo-random function (PRF) under the sole assumption that the internal compression function $h$ (keyed 
by the chaining variable input) is a PRF. The result can be transposed to HMAC as well under the extra assumption that $h$ is also a PRF when keyed by the message input.

Concerning key recovery attacks, an adversary should not be able to recover the key in less than $2^{k}$ computations for both HMAC and NMAC. In the case of NMAC one can attack the two keys $K_{\text {out }}$ and $K_{\text {in }}$ independently by first finding an internal collision and then using this colliding pair information to brute force the first key $K_{i n}$, and finally the second one $K_{\text {out }}$.

Universal or existential forgery attacks should cost $2^{n}$ computations for a perfectly secure $n$-bit MAC. However, the iterated nature of the hash functions used inside NMAC or HMAC allows a simple existential forgery attack requiring only $2^{l / 2}$ computations [17. Indeed, with $2^{l / 2}$ queries, an adversary can first find an internal collision between two messages $\left(M, M^{\prime}\right)$ of the same length during the first hash call. Then, any extra block $m$ added to both of these two messages will lead again to an internal collision. Thus, the attacker can simply forge a valid MAC by only querying for $M \| m$ and deducing that $M^{\prime} \| m$ will have the same $\mathrm{MAC}$ value.

Concerning distinguishers on HMAC or NMAC, two types have been discussed in the literature: Distinguishing- $\mathrm{R}$ and Distinguishing-H attacks, defined below:

Distinguishing-R. Let $\mathcal{F}_{n}$ be the set of $n$-bit output functions. We denote $F_{K}$ the oracle on which the adversary $\mathcal{A}$ can make queries. The oracle is instantiated either with $F_{K}=\operatorname{HMAC}_{K}$ (with $K$ being a randomly chosen $k$-bit key) or with a randomly chosen function $R_{K}$ from $\mathcal{F}_{n}$. The goal of the adversary is to distinguish between the two cases and its advantage is given by

$$
A d v(\mathcal{A})=\left|\operatorname{Pr}\left[\mathcal{A}\left(\operatorname{HMAC}_{K}\right)=1\right]-\operatorname{Pr}\left[\mathcal{A}\left(R_{K}\right)=1\right]\right|
$$

Obviously the collision-based forgery attack detailed above gives directly a distinguishing-R attack on NMAC and HMAC. Thus, the expected security of HMAC and NMAC against distinguishing- $\mathrm{R}$ attacks is $2^{l / 2}$ computations.

Distinguishing-H. The attacker is given access to an oracle $\mathrm{HMAC}_{K}$ and the compression function of the HMAC oracle is instantiated either with a known dedicated compression function $h$ or with a random chosen function $r$ from $\mathcal{F}_{l}^{b+l}$ (the set of $(b+l)$-bit to $l$-bit functions), which we denote $\operatorname{HMAC}_{K}^{h}$ and $\operatorname{HMAC}_{K}^{r}$ respectively. The goal of the adversary is to distinguish between the two cases and its advantage is given by

$$
A d v(\mathcal{A})=\left|\operatorname{Pr}\left[\mathcal{A}\left(\operatorname{HMAC}_{K}^{h}\right)=1\right]-\operatorname{Pr}\left[\mathcal{A}\left(\operatorname{HMAC}_{K}^{r}\right)=1\right]\right| .
$$

The distinguishing-H notion was introduced by Kim et al. 12] for situations where the attacker wants to check which cryptographic hash function is embedded in HMAC. To the best of our knowledge, the best known generic distinguishing$\mathrm{H}$ attack requires $2^{l}$ computations. 
Related-Key Attacks. At Asiacrypt 2012 [14, a new type of generic distinguishing (distinguishing-R or distinguishing- $\mathrm{H}$ ) and forgery attacks for HMAC was proposed. These attacks are in the related-key model and can apply even to wide-pipe proposals, but they only work for HMAC, and only when a special restrictive criterion is verified: the attacker must be able to force a specific difference between the inner and the outer keys (with the predefined values of opad and ipad in HMAC, this criterion is verified when $k=b$ ). The idea is to compare the cycle length when iterating the HMAC construction on small messages with a key $K$, and the cycle length when iterating with a key $K^{\prime}=K \oplus$ opad $\oplus$ ipad.

Attacks on Instantiations with Concrete Hash Functions. Because of its widespread use in many security applications, HMAC has also been carefully scrutinized when instantiated with a concrete hash function, exploiting weaknesses of some existing hash function. In parallel to the recent impressive advances on hash function cryptanalysis, the community analyzed the possible impact on the security of HMAC when instantiated with standards such as MD4 [19], MD5 [20], SHA-1 22 Or HAVAL. In particular, key-recovery attacks have been found on HMAC-MD4 924] and HMAC-HAVAL 27. Concerning the distinguishing-H notion, one can cite for example the works from Kim et al. [12, Rechberger et al. [18] and Wang et al. 25.

However, to put these attacks in perspective, it is important to know the complexity of generic attacks, that work even with a good hash function.

\section{Cycle Detection for HMAC}

Our new attacks are based on some well-known properties of random functions.

\subsection{Random Mapping Properties on a Finite Set}

Let us consider a random function $f$ mapping $n$ bits to $n$ bits and we denote $N=2^{n}$. We would like to know the structure of the functional graph defined by the successive iteration of this function, for example the expected number of components, cycles, etc. First, it is easy to see that each component will contain a single cycle with several trees linked to it. This has already been studied for a long time, and in particular we recall two theorems from Flajolet and Odlyzko [7.

Theorem 1 ([7, Th. 2]). The expectations of the number of components, number of cyclic points, number of terminal points, number of image points, and number of $k$-th iterate image points in a random mapping of size $N$ have the asymptotic forms, as $N \rightarrow \infty$ :
(i) \# Components: $\frac{1}{2} \log N$
(ii) \# Cyclic nodes: $\sqrt{\pi N / 2}$
(iii) \# Terminal nodes: $e^{-1} N$

(iv) \# Image points: $\left(1-e^{-1}\right) N$

(v) \# k-th iterate images: $\left(1-\tau_{k}\right) N$, with $\tau_{0}=0, \tau_{k+1}=e^{-1+\tau_{k}}$. 
In particular, a random mapping has only a logarithmic number of distinct components, and the number of cyclic points follows the square root of $N$.

By choosing a random starting point $P$ and iterating the function $f$, one will follow a path in the functional graph starting from $P$, that will eventually connect to the cycle of the component in which $P$ belongs, and we call tail length the number of points in this path. Similarly, we call cycle length the number of nodes in the cycle. Finally, the number of points in the non-repeating trajectory from $P$ is called the rho length, and we call $\boldsymbol{\alpha}$-node of the path the node that connects the tail and the cycle.

Theorem 2 ([7, Th. 3]). Seen from a random point in a random mapping of size $N$, the expectations of the tail length, cycle length, rho length, tree size, component size, and predecessors size have the following asymptotic forms:

(i) Tail length $(\lambda): \sqrt{\pi N / 8}$

(ii) Cycle length $(\mu): \sqrt{\pi N / 8}$

(iii) Rho length $(\rho=\lambda+\mu): \sqrt{\pi N / 2}$ (iv) Tree size: $N / 3$

(v) Component size: $2 N / 3$

(vi) Predecessors size: $\sqrt{\pi N / 8}$

One can see that, surprisingly, in a random mapping most of the points tend to be grouped together in a single giant component, and there is a giant tree with a significant proportion of the points. The asymptotic expectation of the maximal features is given by Flajolet and Sedgewick [8].

Theorem 3 ([8, VII.14]). In a random mapping of size $N$, the largest tree has an expected size of $\delta_{1} N$ with $\delta_{1} \approx 0.48$ and the largest component has an expected size of $\delta_{2} N$ with $\delta_{2} \approx 0.7582$.

These statistical properties will be useful to understand the advantage of our attacks. We show the functional graph of a simple random-looking function in Figure 4 in the Appendix.

\subsection{Using Cycle-Detection to Obtain Some Secret Information}

In this article and as in [14, we will study the functional graph structure of a function to derive a distinguisher or obtain some secret information. More precisely, in [14. Peyrin et al. observed that the functional graph structure of HMAC was the same when instantiated with a key $K$ or with a related key $K^{\prime}=$ $K \oplus \operatorname{ipad} \oplus$ opad (note that in order to be able to query this related-key $K^{\prime}$, the key $K$ has to be of size $b$ or $b-1$, which is quite restrictive). This is a property that should not exist for a randomly chosen function and they were able to detect this cycle structure by measuring the cycle length in both cases $K$ and $K^{\prime}$, and therefore obtaining a distinguishing-R attack for HMAC in the related-key model. In practice, the attacker can build and observe the functional graph of HMAC by simply successively querying the previous $n$-bit output as new message input.

In this work, instead of studying the structure of the functional graph of HMAC directly, we will instead study the functional graph of the internal compression 
function $h$ with a fixed message block: we denote $h_{M}(X)=h(X, M)$. We aim to obtain some information on $h_{M}$ that we can propagate outside the HMAC structure. This is therefore perfectly suited for a distinguishing-H attack, which requires the attacker to exhibit a property of $h$ when embedded inside the HMAC construction. We can traverse the functional graph of $h_{M}$ by querying the HMAC oracle with a long message composed of many repetitions of the fixed message block $M$. The issue is now to detect some properties of the functional graph of $h_{M}$ inside HMAC and without knowing the secret key. We explain how to do that in the next section.

\section{Distinguishing-H Attack for Hash-Based MACs}

In the rest of the article, we use the notation $[x]^{k}$ to represent the successive concatenation of $k$ message blocks $x$, with $[x]=[x]^{1}$.

\subsection{General Description}

In order to derive a distinguishing-H attack, we need to do some offline computations with the target compression function $h$ and use this information to compare online with the function embedded in the MAC oracle. We use the structure of the functional graph of $h_{[0]}$ to derive our attack (of course we can choose any fixed message block). We can travel in the graph by querying the oracle using consecutive [0] message blocks. However, since the key is unknown, we do not know where we start or where we stop in this graph. We have seen in the previous section that the functional graph of a random function is likely to have a giant component containing most of the nodes. We found that the cycle size of the giant component of $h_{[0]}$ is a property that can be efficiently tested.

More precisely, we first compute the cycle size of the giant component of $h_{[0]}$ offline; we denote it as $L$. Then, we measure the cycle size of the giant component of the unknown function by querying the MAC oracle with long messages composed of many consecutive [0] message blocks. If no length padding is used in the hash function, this is quite simple: we just compare $\operatorname{MAC}\left([0]^{2^{l / 2}}\right)$ and $\operatorname{MAC}\left([0]^{2^{l / 2}+L}\right)$. With a good probability, the sequence of $2^{l / 2}$ zero block is sufficiently long to enter the cycle, and if the cycle has length $L$, the two MAC outputs will collide.

Unfortunately, this method does not work because the lengths of the messages are different, and thus the last message block with the length padding will be different and prevent the cycle collision to propagate to the MAC output. We will use a trick to overcome this issue, even though the basic method remains the same. The idea is to build a message $M$ going twice inside the cycle of the giant component, so that we can add $L[0]$ message blocks in the first cycle to obtain a message $M_{1}$ and $L$ [0] message blocks in the second cycle to obtain $M_{2}$. This is depicted in Figure 2] $M_{1}$ will cycle $L$ [0] message blocks in the first cycle (red dotted arrows), while $M_{2}$ will cycle $L$ [0] message blocks in the second cycle (blue dashed arrows), and thus they will both have the same length overall. 
To perform the distinguishing-H attack, the adversary simply randomly selects an initial message block $m$ and query $M_{1}=m\left\|[0]^{2^{l / 2}}\right\|[1] \|[0]^{2^{l / 2}+L}$ and $M_{2}=m\left\|[0]^{2^{l / 2}+L}\right\|[1] \|[0]^{2^{l / 2}}$ to the MAC oracle, and deduce that the target function is used to instantiate the oracle if the two MAC values are colliding. The [1] message block is used to quit the cycle and randomize the entry point to return again in the giant component. We give below a detailed attack procedure and complexity analysis.

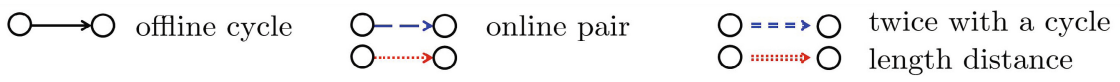

$\alpha_{\nexists}$ offline $\alpha$ node $\quad Q_{0}$ online $\alpha$ node

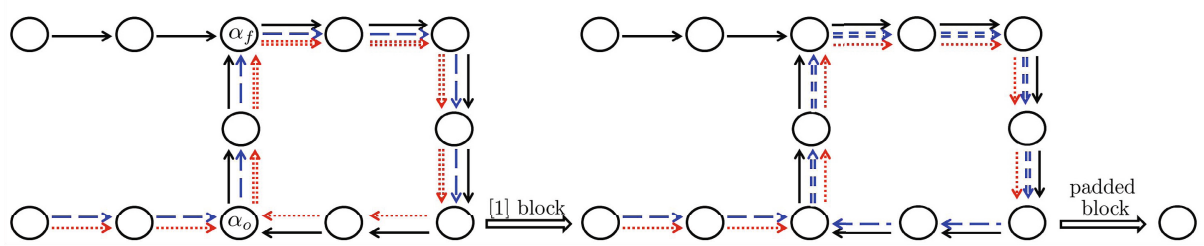

Fig. 2. Distinguishing-H attack

This attack is very interesting as the first generic distinguishing-H attack on HMAC and NMAC with a complexity lower than $2^{l}$. However, we note that the very long message length might be a limitation. In theory this is of no importance and our attack is indeed valid, but in practice some hash functions forbid message inputs longer than a certain length. To address this issue we provide an alternative attack in the full version of this paper, using shorter messages, at the cost of a higher complexity.

\subsection{Detailed Attack Process}

1. (offline) Search for a cycle in the functional graph of $h_{[0]}$ and denote $L$ its length.

2. (online) Choose a random message block $m$ and query the HMAC value of the two messages $M_{1}=m\left\|[0]^{2^{l / 2}}\right\|[1] \|[0]^{2^{l / 2}+L}$ and $M_{2}=m\left\|[0]^{2^{l / 2}+L}\right\|[1] \|[0]^{2^{l / 2}}$.

3. If the HMAC values of $M_{1}$ and $M_{2}$ collide then output 1 , otherwise output 0.

\subsection{Complexity and Success Probability Analysis}

We would like to evaluate the complexity of the attack. The first step will require about $2^{l / 2}$ offline computations to find the cycle for the target compression function $h$. It is important to note that we can run this first step several times 
in order to ensure that we are using the cycle from the largest component of the functional graph of $h_{[0]}$. The second step makes two queries of about $2^{l / 2}+$ $2^{l / 2}+L \simeq 3 \cdot 2^{l / 2}$ message blocks each. Therefore, the overall complexity of the attack is about $2^{l / 2+3}$ compression function computations.

Next, we evaluate the advantage of the adversary in winning the distinguishing-H game defined in Section 2.2. We start with the case where the oracle is instantiated with the real compression function $h$. The adversary will output 1 if a collision happens between the HMAC computations of $M_{1}$ and $M_{2}$. Such a collision can happen when the following conditions are satisfied:

- The processing of the random block $m$ sets us in the same component (of the functional graph of $\left.h_{[0]}\right)$ as in the offline computation. Since we ensured that the largest component was found during the offline computation, and since it has an average size of $0.7582 \cdot 2^{l}$ elements according to Theorem 2 , this event will happen with probability 0.7582 .

- The $2^{l / 2}[0]$ message blocks concatenated after $m$ are enough to reach the cycle of the component, i.e. the tail length when reaching the first cycle is smaller than $2^{l / 2}$. Since the average tail length is less than $2^{l / 2}$ elements, this will happen with probability more than $1 / 2$.

- The processing of the block [1] sets us in the same component (of the functional graph of $h_{[0]}$ ) as in the offline computation; again the probability is 0.7582 .

- The $2^{l / 2}[0]$ message blocks concatenated after [1] are enough to reach the cycle of the component; again this happens with probability $1 / 2$.

The collision probability is then $(0.7582)^{2} \times 1 / 4 \simeq 0.14$ and thus we have that $\operatorname{Pr}\left[\mathcal{A}\left(\operatorname{HMAC}_{K}^{h}\right)=1\right] \geq 0.14$. In the case where the oracle is instantiated with a random function $r$, the adversary will output 1 if and only if a random HMAC collision happens between messages $M_{1}$ and $M_{2}$. Such a collision can be obtained if the processing of any of the last $[0]^{2^{l / 2}}$ blocks of the two messages leads to an internal collision, therefore with negligible probability $2^{l / 2} \cdot 2^{-l}=2^{-l / 2}$. Overall, the adversary advantage is equal to $\operatorname{Adv}(\mathcal{A})=\left|0.14-2^{-l / 2}\right| \simeq 0.14$.

\section{Internal State Recovery for NMAC and HMAC}

This section extends the distinguishing- $\mathrm{H}$ attack in order to build an internal state recovery attack.

\subsection{General Description}

In order to extent the distinguishing-H attack to a state recovery attack, we observe that there is a high probability that the $\alpha$-node reached in the online phase is the root of the giant tree of the functional graph of $h_{[0]}$. More precisely, we can locate the largest tree and the corresponding $\alpha$-node in the offline phase, by repeating the cycle search a few times. We note that $\delta_{1}+\delta_{2}>1$, therefore the largest tree is in the largest component with asymptotic probability one 
(see Theorem 3). Thus, assuming that the online phase succeeds, the $\alpha$-node reached in the online phase is the root of the largest tree with asymptotic probability $\delta_{1} / \delta_{2} \approx 0.63$. If we can locate the $\alpha$-node of the online phase (i.e. we deduce its block index inside the queried message), we immediately get its corresponding internal state value from the offline computations.

Since the average rho length is $\sqrt{\pi 2^{l} / 2}$ we can not use a brute-force search to locate the $\alpha$-node, but we can use a binary search instead. We denote the length of the cycle of the giant component as $L$, and the follow the distinguishing- $H$ attack to reach the cycle. We choose a random message block $m$ and we query the two messages $M_{1}=m\left\|[0]^{2^{l / 2}}\right\|[1] \|[0]^{2^{l / 2}+L}$ and $M_{2}=m\left\|[0]^{2^{l / 2}+L}\right\|[1] \|[0]^{2^{l / 2}}$. If the MAC collide, we know that the state reached after processing $m \|[0]^{2^{l / 2}}$ is located inside the main cycle. We use a binary search to find the smallest $X$ so that the state reached after $m \|[0]^{X}$ is in the cycle.

The first step of the binary search should decide whether the node reached after $m \|[0]^{2^{l / 2-1}}$ is also inside the first online cycle or not. More precisely, we check if the two messages $M_{1}^{\prime}=m\left\|[0]^{2^{l / 2-1}}\right\|[1] \|[0]^{2^{l / 2}+L}$ and $M_{2}^{\prime}=$ $m\left\|[0]^{2^{l / 2-1}+L}\right\|[1] \|[0]^{2^{l / 2}}$ also give a colliding tag. If it is the case, then the node after processing $m \| 2^{l / 2-1}$ is surely inside the cycle, which implies that the $\alpha$-node is necessarily located in the first $2^{l / 2-1}$ zero blocks. On the other hand, if the tags are not colliding, we cannot directly conclude that the $\alpha$-node is located in the second half since there is a non-negligible probability that the $\alpha$-node is in the first half but the [1] block is directing the paths in $M_{1}^{\prime}$ and $M_{2}^{\prime}$ to distinct components in the functional graph of $h_{[0]}$ (in which case the tag are very likely to differ). Therefore, we have to test for collisions with several couples $M_{1}^{u}=m\left\|[0]^{2^{l / 2-1}}\right\|[u]\left\|[0]^{2^{l / 2}+L}, M_{2}^{u}=m\right\|[0]^{2^{l / 2-1}+L}\|[u]\|[0]^{2^{l / 2}}$, and if none of them collide we can safely deduce that the $\alpha$-node is located in the second $2^{l / 2-1}$ zero blocks. Overall, one such step reduces the number of the candidate nodes by a half and we simply continue this binary search in order to eventually obtain the position of the $\alpha$-node with $\log _{2}\left(2^{l / 2}\right)=l / 2$ iterations.

\subsection{Detailed Attack Process}

1. (offline) Search for a cycle in the functional graph of $h_{[0]}$ and denote $L$ its length.

2. (online) Find a message block $m$ such that querying the two messages $M_{1}=$ $m\left\|[0]^{2^{l / 2}}\right\|[1] \|[0]^{2^{l / 2}+L}$ and $M_{2}=m\left\|[0]^{2^{l / 2}+L}\right\|[1] \|[0]^{2^{l / 2}}$ leads to the same HMAC output. Let $X_{1}$ and $X_{2}$ be two integer variables, initialized to the values 0 and $2^{l / 2}$ respectively.

3. (online) Let $X^{\prime}=\left(X_{1}+X_{2}\right) / 2$. Select $\beta \log (l)$ distinct message blocks $[u]$, and for each of them query the HMAC output for messages $M_{1}^{u}=m\left\|[0]^{X^{\prime}}\right\|$ $[u] \|[0]^{2^{l / 2}+L}$ and $M_{2}^{u}=m\left\|[0]^{X^{\prime}+L}\right\|[u] \|[0]^{2^{l / 2}}$. If at least one of the $\left(M_{1}^{u}\right.$, $M_{2}^{u}$ ) pairs leads to a colliding HMAC output, then set $X_{2}=X^{\prime}$. Otherwise, set $X_{1}=X^{\prime}$. We use $\beta=4.5$ as explained later.

4. (online) If $X_{1}+1=X_{2}$ holds, output $X_{2}$ as the block index of the $\alpha$-node. Otherwise, go back to the previous step. 


\subsection{Complexity and Success Probability Analysis}

Complexity. We would like to evaluate the complexity of the attack. The first step will require about $2^{l / 2}$ offline computations to find the cycle. Again, it is important to note that we can run this first step several times in order to ensure that we are using the cycle from the biggest component of the functional graph of $h_{[0]}$. The second step repeats the execution of the distinguishing-H attack from Section 4, which requires $6 \cdot 2^{l / 2}$ computations for a success probability of 0.14 , until it finds a succeeding message block $m$. Therefore, after trying a few $m$ values, we have probability very close to 1 to find a valid one. The third and fourth steps will be executed about $l / 2$ times (for the binary search), and each iteration of the third step performs $2 \cdot \beta \log (l)$ queries of about $2^{l / 2}+2^{l / 2}+L \simeq$ $3 \cdot 2^{l / 2}$ message blocks each. Therefore, the overall complexity of the attack is about $3 \beta \cdot l \cdot \log (l) \cdot 2^{l / 2}$ compression function computations.

Success Probability. Next we evaluate the success probability that the attacker recovers the internal state and this depends on the success probability of the binary search steps. We start with the case where the node after $m \|[0]^{X^{\prime}}$ is inside the first online cycle. The third step will succeed as long as at least one of the $\left(M_{1}^{u}, M_{2}^{u}\right)$ pairs collide on the output (we can omit the false positive collisions which happen with negligible probability). One pair $\left(M_{1}^{u}, M_{2}^{u}\right)$ will indeed collide if:

- The random block $[u]$ sends both messages to the main component of the functional graph of $h_{[0]}$. Since it has an expected size of $\delta_{2} \cdot 2^{l}$ (see Theorem[3), this is the case with probability $\delta_{2}^{2}$.

- The $2^{l / 2}[0]$ message blocks concatenated after $[u]$ are enough to reach the cycle, i.e. the tail length when reaching the second cycle is smaller than $2^{l / 2}$. Since the average tail length is smaller than $2^{l / 2}$ elements, this will happen with probability $1 / 2$ for each message.

After trying $\beta \log (l)$ pairs, the probability that at least one pair collides is $1-$ $\left(1-\delta_{2}^{2} / 4\right)^{\beta \log (l)}$. If we use $\beta=-1 / \log \left(1-\delta_{2}^{2} / 4\right) \approx 4.5$, this gives a probability of $1-1 / l$. On the other hand, if the node after $m \|[0]^{X^{\prime}}$ is not inside the cycle, the third step will succeed when no random collision occurs among the $\beta \log (l)$ tries, and such collisions happen with negligible probability. Overall, since there are $l / 2$ steps in the binary search, the average success probability of the binary search is $(1-1 / l)^{l / 2} \geq e^{-1 / 2} \approx 0.6$.

Finally, the attack succeeds if the $\alpha$-node is actually the root of the giant tree, as computed in the offline phase. This is the case with probability $\delta_{1} / \delta_{2}$, and the success probability of the full state recovery attack is $\delta_{1} / \delta_{2} \cdot e^{-1 / 2} \approx 0.38$.

\section{Internal State Recovery with Shorter Messages}

In the full version of the paper, we give a an alternative internal-state recovery attack using shorter messages, that can also be used as a distinguishing- $\mathrm{H}$ attack 
with shorter messages. The attacks of Sections 4 and 5 have a complexity of $O\left(2^{l / 2}\right)$ using a small number of messages of length $2^{l / 2}$; on the other hand the alternative attack has a complexity $O\left(2^{3 l / 4}\right)$ using $2^{l / 2}$ messages of length $2^{l / 4}$. More generally, if the message size is limited to $2^{s}$ blocks $(s \leq l / 4)$, then the attack requires $2^{l-2 s}$ messages. Due to space constraints, we only give a brief description of this attack here.

\subsection{Entropy Loss in Collisions Finding}

While the previous attacks are based on detecting cycles in the graph of a random function, this alternative attack is based on the fact that finding collisions by iterating a random function does not give a random collision: some particular collisions are much more likely to be found than others. This fact is well known in the context of collision search; for instance van Oorschot and Wiener [23] recommend to randomize the function regularly when looking for a large number of collisions. In this attack, we exploit this property to our advantage: first we use a collision finding algorithm to locate collisions in $h_{M}$ with a fixed M; then we query the MAC oracle with messages with long repetitions of the block $M$ and we detect collisions in the internal state; since the collisions found in this way are not randomly distributed, there is a good probability that we will reach one the collisions that was previously detected in the offline phase.

Actually, the attacks of Sections 4 and 5 can also be seen as following this stategy: we use a collision finding algorithm based on cycle detection (following Pollard's rho algorithm), and we know that with a good probability, the collision found will be the root of the giant tree. For the alternative attack, we use a collision finding algorithm similar to [23], but using using fixed length chains. In the full version of the paper, we study the entropy of the distribution of collisions found in this way, and we show that when using chains of length $2^{s}$, we need about $2^{l / 2-s}$ collisions in order to have a match between the online and offline steps. This translates to an attack complexity of $2^{l-s}$, with $s \leq l / 4$.

\section{Key Recovery for HMAC Based on a Hash Function with an Internal Checksum}

In this section we study HMAC used with a hash function with an internal checksum, such as GOST. We first show that the checksum does not prevent the distinguishing- $\mathrm{H}$ and state recovery attack, but more surprisingly the checksum actually allows to mount a full key-recovery attack significantly more efficient than exhaustive search.

A hash function with an internal checksum computes the sum of all message blocks, and uses this sum as an extra input to the finalization function. The sum can be computed for any group operation, but it will usually be an XOR sum or a modular addition. We use the XOR sum $\operatorname{Sum}^{\oplus}$ to present our attack, but it is applicable with any group operation. 
The checksum technique has been used to enhance the security of a hash function, assuming that controlling the checksum would be an additional challenge for an adversary. While previous work argued its effectiveness [10, our result reveals a surprising fact that incorporating a checksum into a hash function could even weaken its security in some applications such as HMAC.

A notable example of a hash function with a checksum is the GOST hash function, which has been standardized by the Russian Government 6] and by IETF [5]. HMAC-GOST has also been standardized by IETF [15] and is implemented in OpenSSL. GOST uses parameters $n=l=b$, and uses a separate call to process the message length, as follows:

$$
\begin{aligned}
& x_{0}=I V \quad x_{i+1}=h\left(x_{i}, m_{i}\right) \quad x_{*}=h\left(x_{p},|M|\right) \\
& \sigma_{0}=0 \quad \sigma_{i+1}=\sigma_{i} \oplus m_{i} \quad \text { hash }=g\left(x_{*}, \sigma_{p}\right)
\end{aligned}
$$

If this section we describe the attack on GOST-like functions following this structure; Figure 3 shows an HMAC computation with a GOST-like hash function. We give more general attacks when the output is computed as $g\left(x_{p},|M|, \sigma_{p}\right)$ in the full version of this paper.

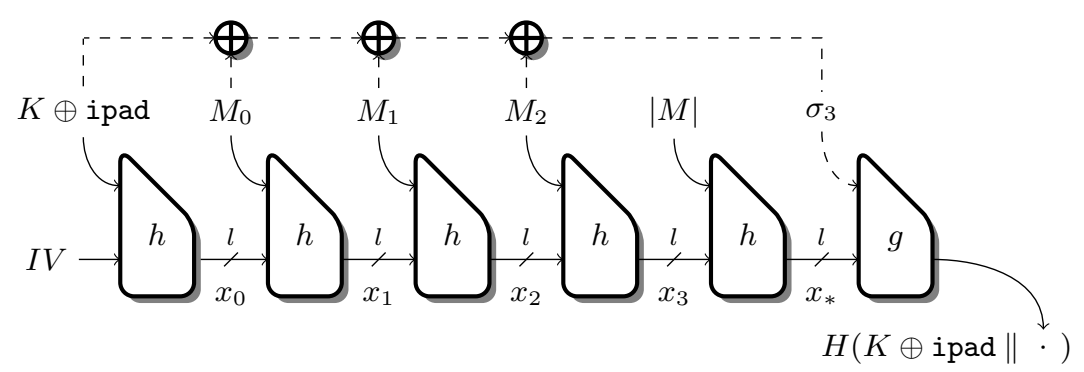

Fig. 3. HMAC based on a hash function with a checksum (dashed lines) and a lengthpadding block. We only detail the first hash function call.

\subsection{General Description}

In HMAC, $K \oplus$ ipad is prepended to a message $M$, and ( $K \oplus$ ipad) $\| M$ is hashed by the underlying hash function $H$. Therefore, the final checksum value is $\sigma_{p}=$ $\operatorname{Sum}^{\oplus}((K \oplus$ ipad $) \| M)=K \oplus \operatorname{ipad} \oplus \operatorname{Sum}^{\oplus}(M)$. In this attack, we use the state recovery attack to recover the internal state $x_{*}$ before the checksum is used and we take advantage of the fact that the value $\sigma_{p}$ actually contains the key, but can still be controlled by changing the message. We use this to inject a known difference in the checksum, and to perform a kind of related key attack on the finalization function $g$, even though we have access to a single HMAC key.

More precisely, we use Joux's multicollision attack [11] to generate a large set of messages with the same value $\bar{x}$ for $x_{*}$, but with different values of the checksum. We detect MAC-collisions among those messages, and we assume 
that the collisions happens when processing the checksum of the internal hash function. For each such collision, we have $g\left(\bar{x}, K \oplus \operatorname{ipad} \oplus \operatorname{Sum}^{\oplus}(M)\right)=g(\bar{x}, K \oplus$ ipad $\left.\oplus \operatorname{Sum}^{\oplus}\left(M^{\prime}\right)\right)$, and we compute the input difference $\Delta M=\operatorname{Sum}^{\oplus}(M) \oplus$ $\operatorname{Sum}^{\oplus}\left(M^{\prime}\right)$.

Finally, we compute $g(\bar{x}, m)$ offline for a large set of random values $m$, and we collect collisions. Again, we compute the input difference $\Delta m=m \oplus m^{\prime}$ for each collision, and we match $\Delta m$ to the previously stored $\Delta M$. When a match is found between the differences we look for the corresponding values and we have $K \oplus$ ipad $\oplus \operatorname{Sum}^{\oplus}(M)=m$ (or $m^{\prime}$ ) with high probability. This gives the value of the key $K$.

State Recovery with a Checksum. First, we note that the checksum $\sigma$ does not prevent the state recovery attacks of Section 5. the complexity only depend on the size $l$ of the state $x$. Indeed, the attack of Section 5 is based on detecting collisions between pairs of messages $M_{1}=m\left\|[0]^{2^{l / 2}}\right\|[k] \|[0]^{2^{l / 2}+L}$ and $M_{2}=m\left\|[0]^{2^{l / 2}+L}\right\|[k] \|[0]^{2^{l / 2}}$. Since the messages have the same checksum, a collision in the state will be preserved. More generally, the attacks can easily be adapted to use only message with a fixed sum. For instance, we can use random messages with two identical blocks in the attack of Section 5] and messages of the form $m\|m\|[0]^{2^{l / 2}}\|[k]\|[k] \|[0]^{2^{l / 2}+L}$ have a checksum of zero.

Recovering the State of a Short Message. Unfortunately, the state we recover will correspond to a rather long message (e.g. $2^{l / 2}$ blocks), and all the queries based on this message will be expensive. In order to overcome this issue, we use the known state $x_{M}$ after a long message $M$ to recover the state after a short one. More precisely, we generate a set of $2^{l / 4}$ long messages by appending a random message blocks $m$ twice to $M$. Note that $\operatorname{Sum}^{\oplus}(M\|m\| m)=\operatorname{Sum}^{\oplus}(M)$. Meanwhile, we generate a set of $2^{3 l / 4}$ two-block messages $m_{1} \| m_{2}$, with $m_{1} \oplus m_{2}=$ $\operatorname{Sum}^{\oplus}(M)$. We query these two sets to the HMAC oracle and collect collisions between a long and a short message. We expect that one collision correspond to a collision in the value $x_{*}$ before the finalization function $g$. We can compute the value $x_{*}$ for the long message from the known state $x_{M}$ after processing $M$. This will correspond to the state after processing the message $m_{1} \| m_{2}$ and its padding block, or equivalently, after processing the message $m_{1}\left\|m_{2}\right\|[2]$ (because the length block is processed with the same compression function). We can verify that the state is correctly recovered by generating a collision $m\left\|m, m^{\prime}\right\| m^{\prime}$ offline from the state $x_{*}$, and comparing $\operatorname{HMAC}\left(m_{1}\left\|m_{2}\right\|[2]\|m\| m\right)$ and $\operatorname{HMAC}\left(m_{1}\left\|m_{2}\right\|[2]\left\|m^{\prime}\right\| m^{\prime}\right)$.

\subsection{Detailed Attack Process}

For simplicity of the description, we omit the padding block in the following description, and we refer to the previous paragraphs for the details of how to deal with the padding. 
1. Recover an internal state value $x_{r}$ after processing a message $M_{r}$ through HMAC. Refer to Section 5 for the detailed process.

2. (online) Choose $2^{l / 4}$ one-block random messages $m$, query $M_{r}\|m\| m$ to HMAC and store $m$ and the corresponding tag.

3. (online) Choose $2^{3 l / 4}$ one-block random messages $m$, query $\left(\operatorname{Sum}^{\oplus}\left(M_{r}\right) \oplus\right.$ $m) \| m$ to HMAC and look for a match between the tag value and one of the stored tag values in Step 2.

For a colliding pair $\left(\operatorname{Sum}^{\oplus}\left(M_{r}\right) \oplus m\right) \| m$ and $M_{r}\left\|m^{\prime}\right\| m^{\prime}$, denote $\operatorname{Sum}^{\oplus}\left(M_{r}\right) \oplus$ $m \| m$ as $M_{1}$ and $h\left(h\left(x_{r}, m^{\prime}\right), m^{\prime}\right)$ as $x_{1}$. Generate a collision $h\left(h\left(x_{1}, u\right), u\right)=$ $h\left(h\left(x_{1}, u^{\prime}\right), u^{\prime}\right)$. Query $M_{1}\|u\| u$ and $M_{1}\left\|u^{\prime}\right\| u^{\prime}$ and compare the tags. If they are equal, the internal state after processing $M_{1}$ (before the checksum block) is $x_{1}$.

4. (offline) Generate $2^{3 l / 4}$ messages that all collide on the internal state before the checksum block by Joux's multicollision. More precisely, choose $2^{l / 2}$ random message $m$ and compute $h\left(x_{1}, m\right)$ to find a collision $h\left(x_{1}, m_{1}\right)=$ $h\left(x_{1}, m_{1}^{\prime}\right)=x_{2}$. Then iterate this procedure to find a collision $h\left(x_{i}, m_{i}\right)=$ $h\left(x_{i}, m_{i}^{\prime}\right)=x_{i+1}$ for $i \leq 3 l / 4$. Denote the value of $x_{3 l / 4+1}$ by $\bar{x}$.

5. (online) Query the set of messages in Step 4 to HMAC in order to collect tag collisions. For each collision $M$ and $M^{\prime}$, compute the checksum difference $\Delta M=\operatorname{Sum}^{\oplus}(M) \oplus \operatorname{Sum}^{\oplus}\left(M^{\prime}\right)$, and store $\left(\operatorname{Sum}^{\oplus}(M), \Delta M\right)$.

6. (offline) Choose a set of $2^{3 l / 4}$ one-block random message $m$, compute $g(\bar{x}, m)$ and collect collisions. For each collision $m$ and $m^{\prime}$, compute the difference $\Delta m=m \oplus m^{\prime}$ and match $\Delta m$ to the stored $\Delta M$ at Step 5. If a match is found, mark $\operatorname{Sum}^{\oplus}(M) \oplus \operatorname{ipad} \oplus m$ and $\operatorname{Sum}^{\oplus}(M) \oplus \operatorname{ipad} \oplus m \oplus \Delta m$ as potential key candidates.

7. (offline) filter the correct key from the potential candidates by verifying a valid message/tag pair.

\subsection{Complexity and Success Probability Analysis}

We need to evaluate the complexity of our key recovery attack.

$\begin{array}{llllll}\text { Step 1: } & O\left(l \cdot \log (l) \cdot 2^{l / 2}\right) & \text { Step 2: } & 2^{3 l / 4} & \text { Step 3: } & 2 \cdot 2^{3 l / 4} \\ \text { Step 4: } & 3 l / 4 \cdot 2^{l / 2} & \text { Step 5: } & 3 l / 4 \cdot 2^{3 l / 4} & \text { Step 6: } & 2^{3 l / 4} \\ \text { Step 7: } & O(1) & & & & \end{array}$

Overall, the fifth step dominates the complexity, and the total complexity is about $3 l / 4 \cdot 2^{3 l / 4}$ compression function computations.

Next we evaluate the success probability of our method. The first step succeeds with a probability almost 1 after several trials. Steps 2 and 3 need to guarantee a collision between a long and a short message. Since there are $2^{l}$ pairs, one such collision occurs with a probability of $1-\left(1-2^{-l}\right)^{2^{l}} \approx 1-1 / e \approx 0.63$. The success probability of producing no less than $2^{l / 2}$ collisions at each of steps 5 and 6 is 0.5 since the expected number of collisions is $2^{l / 2}$. Thus the overall success probability is no less than 0.16 . 


\section{Conclusion}

Our results show that the security of HMAC and hash-based MAC above the birthday bound is significantly weaker than previously expected. First, we show that distinguishing- $\mathrm{H}$ and state-recovery attacks are not much harder than a distinguishing-R attack, contrary to previous beliefs. Second, we show that the use of a checksum can allow a key-recovery attack against HMAC with complexity only $\tilde{O}\left(2^{3 l / 4}\right)$. In particular, this attack is applicable to HMAC-GOST, a standardized construction.

We give a comparison of our attacks and previous attack against concrete instances of HMAC in Table 1, showing that some attacks against concrete instances are in fact less efficient than our generic attacks.

As future works, it would be interesting to find other applications of the internal state recovery for HMAC. Moreover, we expect further applications of the analysis of the functional graph, as it might be possible to use other distinguishing properties, such as the tail length, the distance of a node from the cycle, etc.

Acknowledgments. The authors would like to thank the anonymous referees for their helpful comments. Gaëtan Leurent is supported by the ERC project CRASH. Thomas Peyrin and Lei Wang are supported by the Singapore National Research Foundation Fellowship 2012 (NRF-NRFF2012-06).

\section{References}

1. Bellare, M.: New Proofs for NMAC and HMAC: Security Without CollisionResistance. In: Dwork, C. (ed.) CRYPTO 2006. LNCS, vol. 4117, pp. 602-619. Springer, Heidelberg (2006)

2. Bellare, M., Canetti, R., Krawczyk, H.: Keying Hash Functions for Message Authentication. In: Koblitz, N. (ed.) CRYPTO 1996. LNCS, vol. 1109, pp. 1-15. Springer, Heidelberg (1996)

3. Brassard, G. (ed.): CRYPTO 1989. LNCS, vol. 435. Springer, Heidelberg (1990)

4. Damgård, I.: A Design Principle for Hash Functions. In: [3], pp. 416-427

5. Dolmatov, V.: GOST R 34.11-94: Hash Function Algorithm. RFC 5831 (Informational) (March 2010)

6. FAPSI, VNIIstandart: GOST 34.11-94, Information Technology Cryptographic Data Security Hashing Function (1994) (in Russian)

7. Flajolet, P., Odlyzko, A.M.: Random Mapping Statistics. In: Quisquater, J.-J., Vandewalle, J. (eds.) EUROCRYPT 1989. LNCS, vol. 434, pp. 329-354. Springer, Heidelberg (1990)

8. Flajolet, P., Sedgewick, R.: Analytic Combinatorics. Cambridge University Press (2009)

9. Fouque, P.-A., Leurent, G., Nguyen, P.Q.: Full Key-Recovery Attacks on HMAC/NMAC-MD4 and NMAC-MD5. In: Menezes, A. (ed.) CRYPTO 2007. LNCS, vol. 4622, pp. 13-30. Springer, Heidelberg (2007)

10. Gauravaram, P., Kelsey, J.: Linear-XOR and Additive Checksums Don't Protect Damgård-Merkle Hashes from Generic Attacks. In: Malkin, T. (ed.) CT-RSA 2008. LNCS, vol. 4964, pp. 36-51. Springer, Heidelberg (2008) 
11. Joux, A.: Multicollisions in Iterated Hash Functions. Application to Cascaded Constructions. In: Franklin, M. (ed.) CRYPTO 2004. LNCS, vol. 3152, pp. 306-316. Springer, Heidelberg (2004)

12. Kim, J., Biryukov, A., Preneel, B., Hong, S.: On the Security of HMAC and NMAC Based on HAVAL, MD4, MD5, SHA-0 and SHA-1 (Extended Abstract). In: De Prisco, R., Yung, M. (eds.) SCN 2006. LNCS, vol. 4116, pp. 242-256. Springer, Heidelberg (2006)

13. Merkle, R.C.: One Way Hash Functions and DES. In: [3], pp. 428-446

14. Peyrin, T., Sasaki, Y., Wang, L.: Generic Related-Key Attacks for HMAC. In: Wang, X., Sako, K. (eds.) ASIACRYPT 2012. LNCS, vol. 7658, pp. 580-597. Springer, Heidelberg (2012)

15. Popov, V., Kurepkin, I., Leontiev, S.: Additional Cryptographic Algorithms for Use with GOST 28147-89, GOST R 34.10-94, GOST R 34.10-2001, and GOST R 34.11-94 Algorithms. RFC 4357 (Informational) (January 2006)

16. Preneel, B.: HMAC. In: van Tilborg, H.C.A., Jajodia, S. (eds.) Encyclopedia of Cryptography and Security, 2nd edn., pp. 559-560. Springer (2011)

17. Preneel, B., van Oorschot, P.C.: MDx-MAC and Building Fast MACs from Hash Functions. In: Coppersmith, D. (ed.) CRYPTO 1995. LNCS, vol. 963, pp. 1-14. Springer, Heidelberg (1995)

18. Rechberger, C., Rijmen, V.: New Results on NMAC/HMAC when Instantiated with Popular Hash Functions. J. UCS 14(3), 347-376 (2008)

19. Rivest, R.L.: The MD4 Message Digest Algorithm. In: Menezes, A., Vanstone, S.A. (eds.) CRYPTO 1990. LNCS, vol. 537, pp. 303-311. Springer, Heidelberg (1991)

20. Rivest, R.L.: The MD5 message-digest algorithm. Request for Comments (RFC) 1320, Internet Activities Board, Internet Privacy Task Force (April 1992)

21. Tsudik, G.: Message Authentication with One-Way Hash Functions. In: INFOCOM, pp. 2055-2059 (1992)

22. U.S. Department of Commerce, National Institute of Standards and Technology: Secure Hash Standard (SHS) (Federal Information Processing Standards Publication 180-3) (2008), http://csrc.nist.gov/publications/fips/fips180-3/ fips180-3_final.pdf

23. van Oorschot, P.C., Wiener, M.J.: Parallel Collision Search with Cryptanalytic Applications. J. Cryptology 12(1), 1-28 (1999)

24. Wang, L., Ohta, K., Kunihiro, N.: New Key-Recovery Attacks on HMAC/NMACMD4 and NMAC-MD5. In: Smart, N.P. (ed.) EUROCRYPT 2008. LNCS, vol. 4965, pp. 237-253. Springer, Heidelberg (2008)

25. Wang, X., Yu, H., Wang, W., Zhang, H., Zhan, T.: Cryptanalysis on HMAC/NMAC-MD5 and MD5-MAC. In: Joux, A. (ed.) EUROCRYPT 2009. LNCS, vol. 5479, pp. 121-133. Springer, Heidelberg (2009)

26. Yasuda, K.: "Sandwich" Is Indeed Secure: How to Authenticate a Message with Just One Hashing. In: Pieprzyk, J., Ghodosi, H., Dawson, E. (eds.) ACISP 2007. LNCS, vol. 4586, pp. 355-369. Springer, Heidelberg (2007)

27. Yu, H., Wang, X.: Full Key-Recovery Attack on the HMAC/NMAC Based on 3 and 4-Pass HAVAL. In: Bao, F., Li, H., Wang, G. (eds.) ISPEC 2009. LNCS, vol. 5451, pp. 285-297. Springer, Heidelberg (2009) 


\section{A Additional Figures}

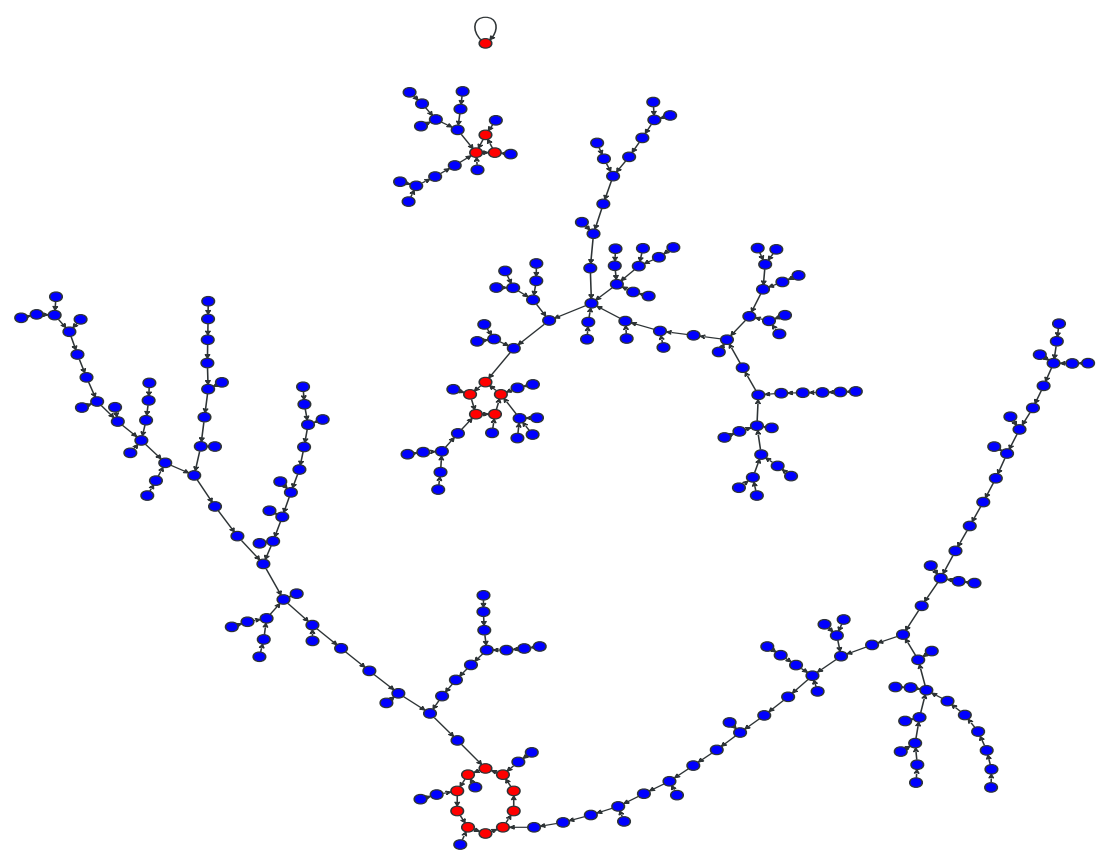

Fig. 4. Functional graph of Keccak (SHA-3) with 8-bit input and 8-bit output 